\section{Desarrollos recientes en la jurisprudencia sobre el derecho a morir}

\author{
ANTONIO BASCUÑÁN R. ${ }^{1, a}$
}

\section{Recent case law about the right to die}

This paper reviews the sentences dictated between 1993 and 2002 by the Supreme Courts of Canada and the Unites States, the House of Lords and Supreme Court of the United Kingdom and the European Human Rights Court, about the validity of the legal prohibition of assistance for suicide. These sentences constituted a judicial consensus about the right to die. This consensus recognized the legal right of patients to reject medical treatments but did not recognize the right to be assisted by a physician to commit suicide. This exclusion is changing in the recent case law of Canada and the United Kingdom, which accepts the fundamental right of terminal patients to medically assisted suicide.

(Rev Med Chile 2016; 144: 483-487)

Key words: Euthanasia, Active; Right to Die; Suicide, Assisted; Value of Life.
'Facultad de Derecho Universidad Adolfo Ibáñez y Universidad de Chile. ${ }^{a}$ Abogado.

El artículo corresponde a una versión resumida de la ponencia del autor titulada "El derecho a morir en la jurisprudencia internacional y constitucional comparada", expuesta en el Primer Seminario Internacional de Bioética y Bioderecho, organizado por la Facultad de Medicina y el Observatorio de Bioética y Bioderecho de la Universidad del Desarrollo y la Clínica Alemana en Santiago los días 12 y 13 de marzo de 2015. Fue elaborado sin una fuente especial de apoyo financiero.

Recibido el 22 de mayo de de 2015, aceptado el 22 de octubre de 2015

Correspondencia a:

Antonio Bascuñán Rodríguez Alcántara 215, Departamento 40 Las Condes.

antonio.bascunan@gmail.com
$\mathrm{E}$ ntre 1993 y 2002 se generó un consenso en relación con el derecho a morir en la jurisprudencia constitucional de Canadá, los Estados Unidos de Norteamérica, el Reino Unido y la jurisprudencia del Tribunal Europeo de Derechos Humanos. A partir del año 2012 es reconocible en Canadá y en el Reino Unido un cambio de perspectiva en la jurisprudencia constitucional sobre la materia. Este artículo ofrece una descripción del consenso previo y de los cambios que están ocurriendo.

\section{El problema constitucional}

Suponiendo que el derecho penal de un país prohíba el auxilio al suicidio o prohíba el homicidio consentido se genera una pregunta constitucional: si la Constitución garantiza un derecho de autonomía personal ¿son estas prohibiciones injustificablemente restrictivas de la autonomía del que desea morir? La respuesta a esta pregunta procede en tres pasos sucesivos: i) ¿Existe un derecho de autonomía personal que abarque la decisión de la persona acerca de cuándo y cómo morir?

ii) ¿Restringen el ejercicio de ese derecho de autonomía las prohibiciones penales del homicidio consentido (en comisión o en comisión por omisión) y del auxilio al suicidio?

iii) ¿Es esa restricción injustificable?

Si se responde afirmativamente las tres preguntas arriba planteadas, la prohibición penal es inconstitucional. La respuesta afirmativa a la pregunta (ii) es obvia: la prohibición legal del auxilio al suicidio y el homicidio consentido declara irrelevante el consentimiento del que va a morir, restringiendo en esa medida su autonomía. Por eso, si se responde afirmativamente a la pregunta (i), entonces todo depende de la respuesta que se dé a la pregunta (iii).

El derecho constitucional de autonomía personal no es el único derecho o principio constitucional que podría entenderse violado por una prohibición legal del auxilio al suicidio 
o del homicidio consentido. Sin embargo, el argumento del derecho de autonomía personal ha sido el más importante y por eso este artículo se concentra en él.

\section{El consenso surgido entre 1993 y 2002}

Lo que caracteriza al consenso es afirmar el derecho del paciente a rechazar el tratamiento médico, incluyendo su interrupción, pero negar el derecho del paciente a obtener del médico el auxilio al suicidio o derechamente la muerte. Es decir, para la situación de la eutanasia pasiva da una respuesta afirmativa a las preguntas (i), (ii) y (iii) del esquema recién expuesto, pero para la situación de la eutanasia activa directa da una respuesta negativa o bien a (i) o bien a (iii).

La primera sentencia de la secuencia que generó este consenso es una decisión de 30 de septiembre de 1993 de la Corte Suprema de Canadá (“CSC”), Rodríguez v. British Columbia ${ }^{1}$. En este caso, Sue Rodríguez una mujer que padecía esclerosis lateral amiotrófica solicitó que se declarara inconstitucional la prohibición del auxilio al suicidio, con el fin de poder recibir el auxilio de un médico cuando decidiera morir, si es que no podía hacerlo por sí misma. La CSC sostuvo que el derecho a decidir acerca de la propia muerte formaba parte del derecho a la seguridad personal garantizado por el art. $7^{\circ}$ de la Carta Canadiense de Derechos y Libertades $^{2}$ y que ese derecho aseguraba un derecho al rechazo del tratamiento, comprensivo de su interrupción, pero que la restricción impuesta a la eutanasia activa por la prohibición del auxilio al suicidio era justificable conforme a los principios fundamentales de justicia.

Las sentencias que siguen en esta secuencia son dos dictadas simultáneamente por la Corte Suprema Federal de los Estados Unidos de Norteamérica ("USSC") el 26 de junio de 1997: Washington v. Glucksberg ${ }^{3}$ y Vacco v. Quill'. En el primer caso, cuatro médicos que ejercían en el Estado de Washington solicitaban que se declarara la inconstitucionalidad de la prohibición del auxilio al suicidio; en el segundo caso solicitaban lo mismo tres médicos que practicaban en el Estado de Nueva York. En ambos casos, las cortes de apelaciones de los respectivos circuitos (Noveno y Segundo) habían acogido la petición de los médicos, lo que fue impugnado por los respectivos estados, que llevaron el caso ante la USSC. En un fallo unánime en la decisión, pero dividido en sus fundamentos, la USSC sostuvo que si bien el derecho a rechazar el tratamiento constituía una libertad específicamente protegida por la cláusula del debido proceso sustantivo de la $14^{\text {a Enmienda }}$ de la Constitución Federal ${ }^{5}$, el interés en ser auxiliado en la propia muerte o ser muerto por otro no tenía ese mismo estatus. Por eso, la prohibición del homicidio consentido y del auxilio al suicidio a lo más podía ser considerada como una restricción de un interés general de libertad, y en esa calidad resultaba plenamente justificable (Glucksberg), también como tratamiento diferenciado bajo la cláusula de la igual protección de la 14a Enmienda de la Constitución Federal (Quill).

La siguiente sentencia es una decisión de 29 de noviembre de 2001 de la Casa de los Lores del Reino Unido ("UKHL"), Pretty v. Director of Public Prosecutions 6 . En este caso, Diane Pretty, una mujer que padecía la enfermedad de la neurona motora, solicitaba que se exigiera judicialmente al Director de Prosecuciones Públicas ("DPP”) del Reino Unido otorgar una declaración que eximiera de responsabilidad penal a su marido por el delito de auxilio al suicidio. La UKHL rechazó por unanimidad la petición, sosteniendo que si bien el derecho inglés reconocía un derecho al rechazo del tratamiento, inclusivo de su interrupción, no reconocía un derecho a ser muerto o auxiliado en el suicidio y que, por lo tanto, el DPP no estaba obligado a dar esa exención. Asimismo, la UKHL sostuvo que el derecho a la vida privada garantizado por el artículo $8^{\circ}$ del Convenio Europeo sobre Derechos Humanos de 1950 ("CEDH") no abarcaba al interés de la persona en decidir acerca de su muerte ${ }^{7}$.

En contra de esta sentencia, Diane Pretty recurrió al Tribunal Europeo de Derechos Humanos ("ECHR"), el cual rechazó por unanimidad su petición en una sentencia de 20 de abril de 2002, inmodificable desde el 29 de julio de 2002, Pretty v. United Kingdom ${ }^{8}$. A diferencia de la UKHL, el ECHR sostuvo que no se podía excluir que el interés en decidir acerca de la propia muerte estuviera protegido por el derecho a la vida privada bajo el $\mathrm{CEDH}$, por lo que la petición era admisible ante el tribunal, pero luego afirmó que la restricción impuesta por la prohibición del auxilio al suicidio y el homicidio consentido eran justificables conforme al número $2^{\circ}$ del artículo $8 \mathrm{CEDH}^{9}$. Esta decisión 
Jurisprudencia sobre el derecho a morir - A. Bascuñán et al

cierra la secuencia de sentencias que generó el consenso jurisprudencial entre 1993 y 2002.

En resumen, analizadas conforme al esquema de tres pasos arriba expuesto, se tiene que la USSC y la UKHL dan una respuesta negativa a la pregunta (i). El ECHR, en cambio, da una respuesta hipotéticamente afirmativa a la pregunta (i) para responder negativamente la pregunta (iii). Por último, la CSC da una respuesta categóricamente afirmativa a la pregunta (i) pero negativa a la pregunta (iii). Los cuatro tribunales coinciden en atribuir al interés del paciente en rechazar el tratamiento médico, incluyendo su interrupción, el estatus de un derecho inequívocamente reconocido por la legislación o la constitución.

\section{El cambio reciente en el Reino Unido}

El cambio de perspectiva del máximo tribunal del Reino Unido comenzó a manifestarse en una sentencia dictada el 30 de julio de 2009, Regina (on the application of Debbie Purdy) $v$ Director of Public Prosecutions ${ }^{10}$. Sufriendo de esclerosis múltiple, Debbie Purdy solicitó información precisa acerca del riesgo que corría su marido de sufrir persecución penal si la acompañaba a morir a Suiza. Siguiendo la decisión del ECHR en Pretty, la UKHL esta vez reconoció que el interés en decidir acerca de la propia muerte está protegido por el derecho a la vida privada del art. $8^{\circ} \mathrm{CEDH}$, y además acogió la apelación, ordenando al DPP publicar una descripción de su política de persecución penal que satisficiera las exigencias expuestas en una de las opiniones que concurrieron al fallo. Tres meses después de esta sentencia, el DPP dio a conocer la nueva regulación, que contempló 16 criterios que hacen más probable la prosecución del auxilio al suicidio y 6 que la hacen menos probable ${ }^{11}$.

La otra sentencia fue dictada el 26 de junio de 2014 por la Corte Suprema del Reino Unido ("UKSC"): Regina (on the application of Nicklinson and another) v. Ministry of Justice, and Regina (on the application of AM) (AP) (Respondent) $v$. The Director of Public Prosecutions ${ }^{12}$. Esta es una decisión compleja. El caso consistió en cuatro apelaciones. Dos de ellas requerían la declaración de incompatibilidad de la prohibición del auxilio al suicidio con el derecho a la vida privada garantizado por el art. $8^{\circ} \mathrm{CEDH}$. Sobre este punto la Corte se dividió de una manera que bien puede ser calificada como paradójica. Por mayoría de siete votos contra dos, la UKSC rechazó las apelaciones. Pero esa mayoría no fue consistente en sus fundamentos, pues cuatro de los votos que concurrieron al rechazo de las apelaciones coincidieron con los dos votos disidentes en un enfoque revolucionario: consideraron que la prohibición general del suicidio asistido es incompatible con el art. $8^{\circ} \mathrm{CEDH}$.

La diferencia entre los cuatro que concurrieron a rechazar las apelaciones y los dos disidentes es una simple consideración temporal. Reconociendo que la declaración de incompatibilidad de la ley con el CEDH es discrecional, esos cuatro jueces consideraron que no era el momento apropiado para la decisión judicial. En otras palabras, decidieron dar una oportunidad al Parlamento inglés, donde se encuentra en tramitación, desde el 6 de junio de 2014, el proyecto de ayuda a morir de Lord Falconer de Thoroton $^{13}$. Pero por lo menos ante esta composición de la UKSC la suerte está echada: o hay nueva legislación que autorice en un margen razonable el auxilio al suicidio o hay declaración de incompatibilidad de su prohibición con el CEDH. Esto implica un giro en 180 grados respecto de Pretty.

\section{El cambio reciente en Canadá}

En la sentencia más extensa de esta secuencia -Carter v. Canada ${ }^{14}$-, la Jueza Lynn Smith de la Corte Suprema de la Columbia Británica -un tribunal de primera instancia, pese a su nombreafirmó la protección constitucional del interés en decidir cuándo y cómo morir como derecho a la seguridad de la persona, afirmó que la prohibición general del suicidio asistido afectaba ese derecho, negó que esa afectación pudiera justificarse conforme a los principios fundamentales de justicia, como lo requiere el art. $7^{\circ}$ de la Carta Canadiense de Derechos y Libertades, y afirmó que la prohibición generaba una diferencia discriminatoria para personas incapacitadas de darse muerte por sí solas. En otras palabras, compartió una de las premisas del voto de mayoría de la CSC en $R o$ dríguez v. British Columbia, pero negó todas las demás premisas. Esto naturalmente genera un problema en un sistema de precedente judicial. La manera en que la Jueza Smith resolvió el problema es institucionalmente plausible: sostuvo que entre 
1993 y 2012 había evolucionado jurisprudencialmente el test de justificación de la afectación de derechos fundamentales conforme a los principios fundamentales de justicia, volviéndose más estricto, y que la justificación de la prohibición por Rodríguez v. British Columbia no satisfacía esos nuevos estándares.

La Corte de Apelaciones de la Columbia Británica revocó la sentencia de la Jueza Smith por consideración al precedente ${ }^{15}$. Pero la CSC acogió por unanimidad la apelación contra la revocación en Carter v. Canada ${ }^{16}$ y declaró que la prohibición general del suicidio asistido infringía injustificablemente el art. 7 de la Carta, en la medida en que prohibía la asistencia médica a la muerte de una persona adulta competente que (i) claramente consiente terminar con su vida y (ii) parece una condición médica grave e irremediable, inclusiva de una discapacidad, que le causa un sufrimiento permanente e intolerable en sus circunstancias. En cuanto al remedio judicial, la Corte rehusó otorgar una excepción constitucional. En su lugar decidió emitir una declaración de invalidez de la ley y suspenderla por 12 meses. El legislador canadiense dispone, pues, de un plazo que vence el próximo 6 de febrero de 2016 si quiere evitar el vacío legal que generará la invalidación de la prohibición del suicidio asistido.

\section{Conclusiones}

No es fácil discernir el efecto de estos cambios en el derecho comparado. Desde luego, hay que esperar a constatar su influencia en la decisión de los respectivos legisladores. Es posible que tanto el Reino Unido como Canadá se unan a los derechos occidentales que han autorizado la eutanasia activa en la forma del auxilio al suicidio. Pero también podría producirse una reacción política adversa a su autorización, que pusiera en entredicho la autoridad de los tribunales para decidir esta cuestión. Eso está por verse.

No hay que exagerar el cambio que traería como consecuencia la consolidación de esta jurisprudencia reciente en la ley canadiense y británica. Aun si ello ocurriera, no cambiaría el tratamiento de todo el ámbito de la eutanasia activa directa, sino sólo del reducido ámbito del auxilio al suicidio y para el caso específico del sufrimiento derivado del padecimiento de patologías o discapacidades incurables. Esto es así, porque la actitud médica hacia el suicidio, al margen del padecimiento de enfermedades graves y el estatus de la prohibición del homicidio consentido, no han sido cuestionadas en Regina (Nicklinson) ni en Carter. El reconocimiento total de la eutanasia activa directa como derecho constitucional del paciente está lejos de esta jurisprudencia.

Pero tampoco hay que menospreciar este cambio reducido. Porque implica un ajuste en la gramática profunda de las distinciones morales y legales relativas a la voluntad de morir. La paradoja del antiguo common law, subyacente a las jurisdicciones canadiense, estadounidense y británica antes del surgimiento del consenso, es que a la vez prohibía el suicidio y autorizaba el rechazo al tratamiento médico. Obviamente, la solución de esta paradoja no podía encontrarse en alguna consideración relativa a la autonomía del paciente, pues el suicidio es la máxima expresión de la voluntad de morir. La solución sólo podía encontrarse en la inviolabilidad del cuerpo humano. La desaparición de la prohibición del suicidio en el common law a mediados del siglo XX generó el primer ajuste de esta gramática profunda, pues removió un obstáculo legal al reconocimiento de la voluntad de morir como expresión de la autonomía. La extensión que ahora presenciamos de la autorización fuerte de la eutanasia pasiva al auxilio al suicidio representa un nuevo ajuste en esta relación entre el principio del respeto a la autonomía y el principio de la inviolabilidad del otro.

El ajuste era inevitable, si es que se fundamenta la prohibición del auxilio al suicidio en la protección de las personas vulnerables por su dudosa competencia o por el riesgo de estar expuestas a presiones, como lo entendieron las sentencias que formaron el consenso entre 1993 y 2002. Porque este no es un fundamento basado en la inviolabilidad del otro, sino en el respeto por su autonomía. Es la clase de paternalismo impropio o débil que resulta consistente con el principio de autonomía. Así fundada la prohibición, su sobreinclusividad es evidente, porque a la vez que protege al paciente que está en riesgo de abuso afecta la autonomía de quien no se encuentra en ese riesgo. En todos los casos en que una voluntad de morir es afirmada de modo serio y libre por un paciente competente, sin riesgo de presiones externas, la prohibición deja de ser para él una protección y se manifiesta como pura restricción de su poder de decisión sobre sí 
mismo. Por eso, era una cuestión de tiempo que esa sobreinclusividad fuera reconocida como lo que es a la luz del principio de autonomía: un problema de exceso estatal.

\section{Referencias}

1. Rodríguez v. British Columbia [1993] 3 S.C.R. 519. http:// scc-csc.lexum.com/scc-csc/scc-csc/en/item/1054/index. do

2. El art. $7^{\circ}$ de la Carta Canadiense de Derechos y Libertades, que es la primera parte de la Ley Constitucional de 1982, dispone: "Todos tienen el derecho a la vida, a la libertad y a la seguridad de la persona y el derecho a no ser deprivado de ello sino de conformidad con los principios fundamentales de justicia" (traducción personal del inglés, disponible en: http://laws-lois.justice.gc.ca/ eng/const/page-15.html).

3. Washington v. Glucksberg 521 U.S. 702 (1997). https:// supreme.justia.com/cases/federal/us/521/702/

4. Vacco v. Quill 521 U.S. 793 (1997). https://supreme. justia.com/cases/federal/us/521/793/

5. En su parte pertinente, la $14^{\mathrm{a}}$ Enmienda de la Constitución federal de los Estados Unidos dispone que "tampoco podrá Estado alguno privar a cualquier persona de la vida, la libertad o la propiedad sin el debido proceso legal; ni negar a cualquier persona que se encuentre dentro de sus límites jurisdiccionales la protección de las leyes, igual para todos" (citada según: http://www. archives.gov/espanol/constitucion.html). La primera oración transcrita consagra la cláusula del debido proceso. La segunda oración consagra la cláusula de la igual protección.

6. Pretty v. Director of Public Prosecutions [2001] UKHL 61. http://www.publications.parliament.uk/pa/ld200102/ ldjudgmt/jd011129/pretty-1.htm

7. El art. $8^{\circ} \mathrm{N}^{\circ} 1 \mathrm{CEDH}$ dispone: “Toda persona tiene derecho al respeto de su vida privada y familiar, de su domicilio y de su correspondencia." http://www. google.cl/url? $\mathrm{sa}=\mathrm{t} \& \mathrm{rct}=\mathrm{j} \& \mathrm{q}=$ \&esrc $=\mathrm{s} \&$ source $=w e-$

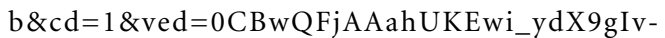
GAhUOLYwKHRjIADE\&url=http\%3A\%2F\%2Fwww. echr.coe.int $\% 2$ FDocuments\%2FConvention_SPA.
pdf\&ei=x0J7Vf_JEY7asASYkIOIAw\&usg $=$ AFQjCNGNd8C0GgNIgbK0w9mxKQyynqMdyQ\&sig2=YAPcbwaGZPjHpw7X5wWxZQ\&bvm=bv.95515949,d.cWc

8. Pretty v. United Kingdom, solicitud $\mathrm{N}^{\circ} 2346 / 2002$, [2002] ECHR 427. http://hudoc.echr.coe.int/sites/eng/pages/search.aspx?i=001-60448\# $\{\% 22$ itemid\%22:[\%22001-60448\%

9. $\mathrm{El}$ art. $8^{\circ} \mathrm{N}^{\circ} 2 \mathrm{CEDH}$ dispone: "No podrá haber injerencia de la autoridad pública en el ejercicio de este derecho sino en tanto en cuanto esta injerencia esté prevista por la ley y constituya una medida que, en una sociedad democrática, sea necesaria para la seguridad nacional, la seguridad pública, el bienestar económico del país, la defensa del orden y la prevención de las infracciones penales, la protección de la salud o de la moral, o la protección de los derechos y las libertades de los demás." http://www.google.cl/url?sa=t\&rct=j\&q=\&esrc=s\&sour-

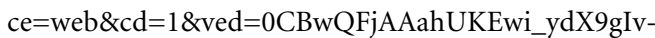
GAhUOLYwKHRjIADE\&url=http\%3A\%2F\%2Fwww. echr.coe.int\%2FDocuments\%2FConvention_SPA. pdf\&ei=x0J7Vf_JEY7asASYkIOIAw\&usg=AFQjCNGN d8C0GgNIgbK0w9mxKQyynqMdyQ\&sig2=YAPcbwaGZPjHpw7X5wWxZQ\&bvm=bv.95515949,d.cWc

10. Regina (on the application of Debbie Purdy) $v$ Director of Public Prosecutions [2009] UKHL 45. http://www.publications.parliament.uk/pa/ld200809/ldjudgmt/jd090730/ rvpurdy.pdf

11. Director of Public Prosecutions, Policy for Prosecutors in Respect of Cases of Encouraging or Assisting Suicide, http://www.cps.gov.uk/publications/prosecution/assisted_suicide_policy.html.

12. Regina (on the application of Nicklinson and another) $v$. Ministry of Justice, and Regina (on the application of AM) (AP) (Respondent) v. The Director of Public Prosecutions [2014] UKSC 38. https://www.supremecourt.uk/decided-cases/docs/UKSC_2013_0235_Judgment.pdf

13. Assisted Dying Bill [HL] 2014-15 (http://services.parliament.uk/bills/2014-15/assisteddying.html).

14. Carter v. Canada [2012] BCSC 88. http://www.canlii. org/en/bc/bcsc/doc/2012/2012bcsc886/2012bcsc886.pdf

15. Carter v. Canada [2013] BCCA 435. http://www.courts. gov.bc.ca/jdb-txt/CA/13/04/2013BCCA0435.htm

16. Carter v. Canada [2015] SCC 5. https://scc-csc.lexum. com/scc-csc/scc-csc/en/item/14637/index.do 